\title{
A Study on the Fundamental Unit of Certain Real Quadratic Number Fields
}

\author{
Özen ÖZER* \\ Department of Mathematics, Faculty of Science and Arts, Kırklareli Üniversity, Kırklareli \\ *Corresponding author: ozenozer39@gmail.com
}

Received October 11, 2017; Revised December 13, 2017; Accepted January 26, 2018

\begin{abstract}
In this paper, we consider the certain types of real quadratic fields $\mathbb{Q}(\sqrt{\mathrm{d}})$ where $\mathrm{d}$ is a square free positive integer. We obtain new parametric representation of the fundamental unit $\varepsilon_{d}$ for such types of fields. Also, we get a fix on Yokoi's invariants as well as class numbers and support all results with tables.
\end{abstract}

Keywords: quadratic fields, continued fraction expansions, class numbers, fundamental units, Yokoi's invariants

Cite This Article: Özen ÖZER, "A Study on the Fundamental Unit of Certain Real Quadratic Number Fields." Turkish Journal of Analysis and Number Theory, vol. 6, no. 1 (2018): 1-8. doi: 10.12691/tjant-6-1-1.

\section{Introduction}

Quadratic fields have many applications to different fields of mathematics which contain algebraic number theory, algebraic geometry, algebra, cryptology, and also other scientific fields like computer science. It is also well known that the fundamental units play an important role in studying the class number problem, unit group, pell equations, cryptology, network security and even computer science.

Recently, in [1], Benamar and his co-authors worked on a type of special monic and non square free polynomials related with fixed period continued fraction expansion of square root of rational integers. In [2], Clemens with collaborators proved explicit continued fractions with almost periodic or almost symmetric patterns in their partial quotients, and infinite series whose terms satisfy certain recurrence relations using Newton's method. Tomita and Kawamato [5] constructed an infinite family of real quadratic fields with large even period of minimal type with class number. Zhang and Yue [22] investigated fundamental unit with positive norm as well as several congruence relations about the coefficient of fundamental unit. Halter-Koch [4] studied on a construction of infinite families of real quadratic fields with large fundamental units. Yokoi defined several invariants which two of them were significant invariants and determined as $m_{d}$ and $n_{d}$ by using the coefficients of fundamental unit $[18,19,20,21]$. The author $[10,11]$, has obtained several important results on fundamental units and Yokoi's invariants for special type of $w_{d}$ in the case of $d \equiv$ $2,3(\bmod 4)$.

We also refer other significant references which were worked on the fundamental unit, prime producing polynomials, class numbers problem, continued fraction expansions etc... to the readers for more information and background about the quadratic fields.
The fundamental unit $\varepsilon_{d}=\left(t_{d}+u_{d} \sqrt{d}\right) / 2>1$ of the ring of algebraic integers in a real quadratic number field $\mathbb{Q}(\sqrt{d})$ is a generator of the group of units. Furthermore, integral basis element of algebraic integer's ring in real quadratic fields is determined by either

$$
w_{d}=\sqrt{d}=\left[a_{0} ; \overline{a_{1}, a_{2}, \ldots, a_{\ell(\mathrm{d})-1}, 2 a_{0}}\right]
$$

in the case of $d \equiv 2,3(\bmod 4)$ or

$$
w_{d}=\frac{1+\sqrt{d}}{2}=\left[a_{0} ; \overline{a_{1}, a_{2}, \ldots, a_{\ell(\mathrm{d})-1}, 2 a_{0}-1}\right]
$$

in the case of $d \equiv 1(\bmod 4)$, where $\ell(\mathrm{d})$ is the period length of continued fraction expansion.

For the set $I(d)$ of all quadratic irrational numbers in $\mathbb{Q}(\sqrt{d})$, we say that $\alpha$ in $I(d)$ is reduced if $\alpha>1,-1<$ $\alpha^{\prime}<0\left(\alpha^{\prime}\right.$ is the conjugate of $\alpha$ with respect to $\left.\mathbb{Q}\right)$, and denote by $R(d)$ the set of all reduced quadratic irrational numbers in $I(d)$. Then, it is well known that any number $\alpha$ in $R(d)$ is purely periodic in the continued fraction expansion and the denominator of its modular automorphism is equal to fundamental unit $\varepsilon_{d}$ of $\mathbb{Q}(\sqrt{d})$. Also, Yokoi's invariants, which were defined by H.Yokoi are determined by the coefficient of fundamental unit $\varepsilon_{d}=\frac{t_{d}+u_{d} \sqrt{d}}{2}$ as $m_{d}=\llbracket \frac{u_{d}^{2}}{t_{d}} \rrbracket$ and $n_{d}=\llbracket \frac{t_{d}}{u_{d}^{2}} \rrbracket$ where $\llbracket x \rrbracket$ represents the greatest integer not greater than $x$ (floor of $x$ ).

Continued fraction expansion of integral basis elemet has got two different forms according to $d$ is a square free positive integer congruent to 2,3 modulo 4 or to congruent 1 modulo 4. Besides, the partial constants of continued fraction expansions create the different forms of $d$ for $\mathbb{Q}(\sqrt{d})$ real quadratic number fields.

The aim of this paper is to classify some types of $\mathbb{Q}(\sqrt{d})$ real quadratic number fields where $d$ is a positive 
square free integer. Such real quadratic fields include the continued fraction expansion of the integral basis element $w_{d}$ which has got partial constant elements are same and written as nines (except the last digit of the period). The representation of fundamental unit is determined for such types of real quadratic fields using the parametrization of positive square free integers $d$ (not only in the case of $d \equiv 2,3(\bmod 4)$ but also in the case of $d \equiv 1(\bmod 4))$. Also, the present paper deals with computing Yokoi's invariants $m_{d}$ and $n_{d}$ as well as class numbers. Using the practical way, the results obtained in this paper are supported by numerical tables.

\section{Prelimineries}

In this section we also give some fundamental concepts for the proof of our main theorems defined in the next section. Definition 2.1. $\left\{K_{i}\right\}$ is called as a sequence defined by the recurrence relation

$$
K_{i}=9 K_{i-1}+K_{i-2}
$$

with the initial conditions $K_{0}=0$ and $K_{1}=1$ for $i \geq 2$.

Lemma 2.2. Let $d$ be a square free positive integer such that $d$ congruent to 1 modulo 4 . If we put $w_{d}=\frac{1+\sqrt{d}}{2}$, $a_{0}=\llbracket w_{d} \rrbracket$ into the $w_{R}=\left(a_{0}-1\right)+w_{d}$, then $w_{d} \notin$ $R(d)$ but $w_{R} \in R(d)$ holds. Moreover, for the period $l=\ell(\mathrm{d})$ of $w_{R}$, we get

$$
\begin{aligned}
& w_{R}=\left[\overline{2 a_{0}-1, a_{1}, \ldots \ldots, a_{l-1}}\right] \\
& \text { and } w_{d}=\left[a_{0}, \overline{a_{1}, \ldots \ldots, a_{l-1}, 2 a_{0}-1}\right] .
\end{aligned}
$$

Let

$$
w_{R}=\frac{\left(P_{l} w_{R}+P_{l-1}\right)}{\left(Q_{l} w_{R}+Q_{l-1}\right)}=\left[\overline{2 a_{0}-1, a_{1}, \ldots \ldots ., a_{l-1}, w_{R}}\right]
$$

be a modular automorphism of $w_{R}$, then the fundamental unit $\epsilon_{d}$ of $\mathbb{Q}(\sqrt{d})$ is given by the formulae

$$
\begin{gathered}
\varepsilon_{d}=\frac{t_{d}+u_{d} \sqrt{d}}{2}, \\
t_{d}=\left(2 a_{0}-1\right) \cdot Q_{\ell(d)}+2 Q_{\ell(d)-1}, u_{d}=Q_{\ell(d)}
\end{gathered}
$$

where $Q_{i}$ is determined by $Q_{0}=0, Q_{1}=1$ and $Q_{i+1}=a_{i} Q_{i}+Q_{i-1},(i \geq 1)$.

Proof. Proof is omitted in [15].

Lemma 2.3. Let $d$ be a square free positive integer congruent to 2,3 modulo 4 . If we put $w_{d}=\sqrt{d}, a_{0}=$ $\llbracket \sqrt{d} \rrbracket$ into the $w_{R}=a_{0}+w_{d}$, then we get $w_{d} \notin R(d)$, but $w_{R} \in R(d)$.

Furthermore, for the period $l=l(d)$ of $w_{R}$, we have $w_{R}=\left[\overline{2 a_{0}, a_{1}, a_{2}, \ldots, a_{l(d)-1}}\right]$ and

$$
w_{d}=\left[a_{0} ; \overline{a_{1}, a_{2}, \ldots, a_{l(d)-1}, 2 a_{0}}\right] \text {. }
$$

Besides, let

$$
w_{R}=\frac{w_{R} P_{l}+P_{l-1}}{w_{R} Q_{l}+Q_{l-1}}=\left[2 a_{0}, a_{1}, a_{2}, \ldots, a_{l(d)-1}, w_{R}\right]
$$

be a modular automorphism of $w_{R}$. Then the fundamental unit $\varepsilon_{d}$ of $\mathbb{Q}(\sqrt{d})$ is given by the following formula:

$$
\begin{gathered}
\varepsilon_{d}=\frac{t_{d}+u_{d} \sqrt{d}}{2}=\left(a_{0}+\sqrt{d}\right) Q_{l(d)}+Q_{l(d)-1} \\
t_{d}=2 a_{0} Q_{l(d)}+2 Q_{l(d)-1} \text { and } u_{d}=2 Q_{l(d)}
\end{gathered}
$$

where $Q_{i}$ is determined by $Q_{0}=0, Q_{1}=1$ and $Q_{i+1}=a_{i} Q_{i}+Q_{i-1}$ for $i \geq 1$.

Proof. Proof can be obtained in a similar way as the proof of Lemma 2.2.

\section{Theorems and Results}

Theorem 3.1. Let $d$ be square free positive integer and $\ell \geq 2$ be a positive integer.

(1) We suppose that

$$
d=\left(2 \xi K_{\ell}+9\right)^{2}+8 \xi K_{\ell-1}+4
$$

where $\xi>0$ is a positive integer. In this case, we obtain that $\mathrm{d} \equiv 1(\bmod 4)$ and

$$
w_{d}=[5+\xi K_{\ell} ; \underbrace{\overline{9,9, \ldots, 9}, 2 \xi K_{\ell}+9}_{\ell-1}]
$$

with $\ell=\ell(d)$. Moreover, we get

$$
t_{d}=2 \xi K_{\ell}^{2}+9 K_{\ell}+2 K_{\ell-1} \text { and } u_{d}=K_{\ell}
$$

for $\varepsilon_{d}=\frac{t_{d}+u_{d} \sqrt{d}}{2}$.

(2) In the case of $\ell \equiv 0(\bmod 3)$, if we assume that

$$
d=\left(9+\xi K_{\ell}\right)^{2}+4 \xi K_{\ell-1}+4
$$

for $\xi>0$ odd positive integer, then $d \equiv 1(\bmod 4)$ and

$$
w_{d}=[5+\frac{\xi K_{\ell}}{2} ; \underbrace{\overline{9,9, \ldots, 9,9+\xi K_{\ell}}}_{\ell-1}] \text {. }
$$

Also, in this case

$$
t_{d}=\xi K_{\ell}^{2}+9 K_{\ell}+2 K_{\ell-1} \text { and } u_{d}=K_{\ell}
$$

hold for $\varepsilon_{d}=\frac{t_{d}+u_{d} \sqrt{d}}{2}$.

Remark 1. it is clear that $K_{\ell}$ is odd number if $\ell \not \equiv 0(\bmod 3)$. In the case of $(2), \frac{\xi K_{\ell}}{2}$ is not integer if we substitue $\xi$ odd positive numbers into the parametrization of $\mathrm{d}$ for $\ell \neq \equiv 0(\bmod 3)$. So, we have to put a condition as $\ell$ is divided by 3 in the case of (2). Also, if we choose $\xi$ is even integer, the parametrization of $d$ coincides with the case of (1). That's why, we have to consider $\ell \equiv 0(\bmod 3)$ and $\xi>0$ positive odd integer in the case of (2).

Proof. (1) For any $\ell \geq 2$ and $\xi>0$ positive integer, $d \equiv 1(\bmod 4)$ holds since $\left(2 \xi K_{\ell}+9\right)$ is odd integer. From Lemma 2.2, we know that $w_{d}=\frac{1+\sqrt{d}}{2}, a_{0}=\llbracket w_{d} \rrbracket$ and $w_{R}=\left(a_{0}-1\right)+w_{d}$.

By using these equations, we obtain 


$$
w_{R}=\left(4+\xi K_{\ell}\right)+[5+\xi K_{\ell} ; \underbrace{\overline{9,9, \ldots, 9}, 9+2 \xi K_{\ell}}_{\ell-1}] .
$$

So, we get

$$
\begin{aligned}
& w_{R}=\left(9+2 \xi K_{\ell}\right)+\frac{1}{9+\frac{1}{9+\frac{1}{\ddots}}} \\
& +\frac{1}{9+\frac{1}{w_{R}}} \\
& =\left(9+2 \xi K_{\ell}\right)+\frac{1}{9}+\cdots+\frac{1}{9}+\frac{1}{w_{R}} .
\end{aligned}
$$

By a straight forward induction argument, we have

$$
w_{R}=\left(9+2 \xi K_{\ell}\right)+\frac{K_{\ell-1} w_{R}+K_{\ell-2}}{K_{\ell} w_{R}+K_{\ell-1}} .
$$

Using Definition 2.1 and rearanging the above equality, we obtain

$$
w_{R}^{2}-\left(9+2 \xi K_{\ell}\right) w_{R}-\left(1+2 \xi K_{\ell-1}\right)=0 .
$$

This implies that $w_{R}=\frac{\left(9+2 \xi K_{\ell}\right)+\sqrt{d}}{2}$ since $w_{R}>0$. If we consider Lemma 2.2, we get

$$
\frac{1+\sqrt{d}}{2}=[5+\xi K_{\ell} ; \underbrace{\overline{9,9, \ldots, 9}, 9+2 \xi K_{\ell}}_{\ell-1}]
$$

and $\ell=\ell(d)$.

Now, we have to determine $\varepsilon_{d}, t_{d}$ and $u_{d}$ using Lemma 2.2 again. It is clear that $Q_{i}=K_{i}$ by induction for $\forall i \geq 0$. If we substitute the values of sequence into the coefficients of fundamental unit

$$
t_{d}=2 \xi K_{\ell}^{2}+9 K_{\ell}+2 K_{\ell-1} \text { and } u_{d}=K_{\ell}
$$

holds for $\varepsilon_{d}=\frac{t_{d}+u_{d} \sqrt{d}}{2}$.

(2) In the case of $\ell \equiv 0(\bmod 3)$, we get $K_{\ell} \equiv 0(\bmod 2)$. By subsituting this equivalence into the parametrization of $d$, we have $d \equiv 1(\bmod 4)$ for $\xi>0$ positive odd integer. By using Lemma 2.2 and the parametrization of $d=$ $\left(9+\xi K_{\ell}\right)^{2}+4 \xi K_{\ell-1}+4$, we have $w_{R}=\left(a_{0}-1\right)+w_{d}$. Then, we have

$$
\begin{aligned}
& w_{R}=\left(4+\frac{\xi K_{\ell}}{2}\right)+[5+\frac{\xi K_{\ell}}{2} ; \underbrace{\overline{9,9, \ldots, 9}, 9+\xi K_{\ell}}_{\ell-1}] \\
& \Rightarrow w_{R}=\left(9+\xi K_{\ell}\right)+\frac{1}{9+\frac{1}{9+\frac{1}{\ddots}}} \\
& =\left(9+\xi K_{\ell}\right)+\frac{1}{9}+\cdots+\frac{1}{9}+\frac{1}{w_{R}} .
\end{aligned}
$$

By a straight forward induction argument, we get

$$
w_{R}=\left(9+\xi K_{\ell}\right)+\frac{K_{\ell-1} w_{R}+K_{\ell-2}}{K_{\ell} w_{R}+K_{\ell-1}} .
$$

Rearranging and using Definition 2.1 into the above equality, we obtain

$$
w_{R}^{2}-\left(9+\xi K_{\ell}\right) w_{R}-\left(1+\xi K_{\ell-1}\right)=0 .
$$

This implies that $w_{R}=\left(4+\frac{\xi K_{\ell}}{2}\right)+\frac{1+\sqrt{d}}{2}$ since $w_{R}>0$. If we consider Lemma 2.2, we get

$$
\frac{1+\sqrt{d}}{2}=[5+\frac{\xi K_{\ell}}{2} ; \underbrace{\overline{9,9, \ldots, 9}, 9+\xi K_{\ell}}_{\ell-1}]
$$

and $\ell=\ell(d)$.

Using $Q_{i}=K_{i}$ for $\forall i \geq 0$, we obtain the coefficients of fundamental unit $t_{d}=\xi K_{\ell}^{2}+9 K_{\ell}+2 K_{\ell-1}$ and $u_{d}=K_{\ell}$ for $\varepsilon_{d}=\frac{t_{d}+u_{d} \sqrt{d}}{2}$.

Corollary 3.2. Let $d$ be a square free positive integer congruent to 1 modulo 4 . If we assume that $d$ is satisfying the conditions in Theorem 3.1, then it always hold Yokoi's invariant $m_{d}=0$.

Proof. $m_{d}$ is defined $m_{d}=\llbracket \frac{u_{d}^{2}}{t_{d}} \rrbracket$ by Yokoi. In the case of (1) if we substitue $t_{d}$ and $u_{d}$ into the $m_{d}$, then we obtain,

$$
m_{d}=\llbracket \frac{u_{d}^{2}}{t_{d}} \rrbracket=\llbracket \frac{K_{\ell}^{2}}{2 \xi K_{\ell}^{2}+9 K_{\ell}+2 K_{\ell-1}} \rrbracket .
$$

So, we get $m_{d}=0$ since $t_{d}>u_{d}^{2}$ for $\xi>0$ positive integer.

In a similar way, we obtain

$$
m_{d}=\left\|\frac{u_{d}^{2}}{t_{d}}\right\|=\left\|\frac{K_{\ell}^{2}}{\xi K_{\ell}^{2}+9 K_{\ell}+2 K_{\ell-1}}\right\|=0
$$

since $t_{d}>u_{d}^{2}$ for $\xi>0$ positive odd integer in the case of (2).

Corollary 3.3. Let $d$ be the square free positive integer positive integer corresponding to $\mathbb{Q}(\sqrt{d})$ holding (1) in the Theorem 3.1. We tabulate the Table 3.1, where fundamental unit is $\varepsilon_{d}$, integral basis element is $w_{d}$ and Yokoi's invariant is $n_{d}$ for $\xi=1,2$ and $2 \leq \ell(d) \leq 10$.

Proof. This Corollary is obtained from Theorem 3.1 by taking $\xi=1$ or 2 in the case of (1). $n_{d}$ is defined as $n_{d}=\llbracket \frac{t_{d}}{u_{d}^{2}} \rrbracket$. If we substitue $t_{d}$ and $u_{d}$ into the $n_{d}$, then we get

$$
\begin{aligned}
& n_{d}=\llbracket \frac{t_{d}}{u_{d}^{2}} \rrbracket=\llbracket \frac{2 K_{\ell}^{2}+9 K_{\ell}+2 K_{\ell-1}}{K_{\ell}^{2}} \rrbracket \\
& =2+\llbracket \frac{9 K_{\ell}+2 K_{\ell-1}}{K_{\ell}^{2}} \rrbracket
\end{aligned}
$$

for $\xi=1$. Also, we get $n_{d}=3$ for $\ell=2$. Since $K_{\ell}$ is increasing sequence, we obtain

$$
2,113>\left(\frac{2 K_{\ell}^{2}+9 K_{\ell}+2 K_{\ell-1}}{K_{\ell}^{2}}\right)>2
$$

for $\ell \geq 3$. So, we have $n_{d}=2$ for $\ell \geq 3$. Besides, in the case of $\xi=2$, we get $n_{d}=5$ for $\ell=2$ as well as $n_{d}=4$ for $\ell \geq 3$ by using similar way. The proof of Corollary 3.3 is completed. 
Table 3.1. Square-free positive integers $d$ with $2 \leq \ell(d) \leq 10$ (In this table, we rule out $\ell(d)=4$ for $\xi=2$ since $d$ is not a square free positive integer.)

\begin{tabular}{|l|c|c|c|c|c|}
\hline \multicolumn{1}{|c|}{$\boldsymbol{\xi}$} & $\boldsymbol{\ell}$ & $\boldsymbol{\ell}(\boldsymbol{d})$ & $\boldsymbol{n}_{\boldsymbol{d}}$ & $\boldsymbol{w}_{\boldsymbol{d}}$ & $\boldsymbol{\varepsilon}_{\boldsymbol{d}}$ \\
\hline 741 & 1 & 2 & 3 & {$[14 ; \overline{9,27}]$} & $(245+9 \sqrt{741}) / 2$ \\
\hline 30005 & 1 & 3 & 2 & {$[87 ; \overline{9,9,173}]$} & $(14204+82 \sqrt{30005}) / 2$ \\
\hline 2259669 & 1 & 4 & 2 & {$[752 ; \overline{9,9,9,1503}]$} & $(92678789+6805 \sqrt{185483141}) / 2$ \\
\hline 185483141 & 1 & 5 & 2 & {$[6810 ; \overline{9, \ldots, 9,13619}]$} & $(7686587666+61992 \sqrt{15374318493) / 2}$ \\
\hline 15374318493 & 1 & 6 & 2 & {$[61997 ; \overline{9, \ldots, 9,123993}]$} & $(637851929159+564733 \sqrt{1275714271565}) / 2$ \\
\hline 1275714271565 & 1 & 7 & 2 & {$[564738 ; \overline{9, \ldots, 9,1129475}]$} & $\left(\begin{array}{c}52933639388609+ \\
5144589 \sqrt{105867373638837}) / 2\end{array}\right.$ \\
\hline 105867373638837 & 1 & 8 & 2 & {$[5144594 ; \overline{9, \ldots, 9,10289187}]$} & $\left(\begin{array}{c}4392850717861796+ \\
46866034 \sqrt{8785702299890645}) / 2\end{array}\right.$ \\
\hline 8785702299890645 & 1 & 9 & 2 & {$[46866039 ; \overline{9, \ldots, 9,93732077}]$} & $\left(\begin{array}{c}364553644063824173+ \\
426938895 \sqrt{729107296000012677}) / 2\end{array}\right.$ \\
\hline 729107296000012677 & 1 & 10 & 2 & {$[426938900 ; \overline{9, \ldots, 9,853877799}]$} & $(407+9 \sqrt{2045}) / 2$ \\
\hline 2045 & 2 & 2 & 5 & {$[23 ; \overline{9,45}]$} & $(27652+82 \sqrt{113717}) / 2$ \\
\hline 113717 & 2 & 3 & 4 & {$[169 ; \overline{9,9,337}]$} & $(185294839+6805 \sqrt{741430397}) / 2$ \\
\hline 741430397 & 2 & 5 & 4 & {$[13615 ; \overline{9, \ldots, 9,27229}]$} & $(15372603794+701 \sqrt{61492701413}) / 2$ \\
\hline 61492701413 & 2 & 6 & 4 & {$[123989 ; \overline{9, \ldots, 9,247977}]$} & $(1275698651737+564733 \sqrt{5102815433357}) / 2$ \\
\hline 5102815433357 & 2 & 7 & 4 & {$[1129471 ; \overline{9, \ldots, 9,2258941}]$} & $\left(\begin{array}{c}105867231346451+ \\
5144589 \sqrt{423469115108957}) / 2\end{array}\right.$ \\
\hline 423469115108957 & 2 & 8 & 4 & {$[10289183 ; \overline{9, \ldots, 9,20578365}]$} & $8785701003640108+$ \\
\hline 35142805742894453 & 2 & 9 & 4 & {$[93732073 ; \overline{9, \ldots, 9,187464145}]$} & $(4666034 \sqrt{35142805742894453}) / 2$ \\
\hline 2916429152510593469 & 2 & 10 & 4 & {$[853877795 ; \overline{9, \ldots, 9,1707755589}]$} & $\left(\begin{array}{c}729107284191466223+ \\
426938895 \sqrt{2916429152510593469}) / 2\end{array}\right.$ \\
\hline
\end{tabular}

Remark 2. In the Table 3.1, using the classical Dirichlet class number formula, we calculate class number $h_{d}=2$ for both the real quadratic field $\mathbb{Q}(\sqrt{741})$ and $\mathbb{Q}(\sqrt{2045})$. These fields are also obtained in the Table 2.1 of reference [8]. Additionaly, using the classical Dirichlet class number formula and computer calculations, we can see the other class numbers for several real quadratic fields in the |Table 3.1 as follows:

\begin{tabular}{|c|c|}
\hline $\boldsymbol{d}$ & $\boldsymbol{h}_{\boldsymbol{d}}$ \\
\hline 741 & 2 \\
\hline 30005 & 4 \\
\hline 2259669 & 36 \\
\hline 185483141 & 294 \\
\hline 2045 & 2 \\
\hline 113717 & 7 \\
\hline 741430397 & 383 \\
\hline
\end{tabular}

Corollary 3.4. Let $d$ be the square free positive integer positive integer corresponding to $\mathbb{Q}(\sqrt{d})$ holding (2) in Theorem 3.1. We state the Table 3.2 where fundamental unit is $\varepsilon_{d}$, integral basis element is $w_{d}$ and Yokoi's invariant is $n_{d}$ for $\xi=1,3$ and $3 \leq \ell(d) \leq 12$.

Proof. By subsituting $\xi=1$ or 3 into the (2) of Theorem 3.1 , we get this corallary and the table. If we substitue $t_{d}$ and $u_{d}$ into the $n_{d}=\llbracket \frac{t_{d}}{u_{d}^{2}} \rrbracket$, then we have

$$
n_{d}=\llbracket \frac{t_{d}}{u_{d}^{2}} \rrbracket=\llbracket \frac{K_{\ell}^{2}+9 K_{\ell}+2 K_{\ell-1}}{K_{\ell}^{2}} \rrbracket=1+\llbracket \frac{9 K_{\ell}+2 K_{\ell-1}}{K_{\ell}^{2}} \rrbracket
$$

for $\xi=1$. Since $K_{\ell}$ is increasing sequence, we obtain

$$
1,113>\left(\frac{K_{\ell}^{2}+9 K_{\ell}+2 K_{\ell-1}}{K_{\ell}^{2}}\right)>1
$$

for $\ell \geq 3$. Therefore, we obtain $n_{d}=1$ for $\ell \geq 3$. Also, we get $n_{d}=3$ since

$$
3,113>\left(\frac{3 K_{\ell}^{2}+9 K_{\ell}+2 K_{\ell-1}}{K_{\ell}^{2}}\right)>3
$$

for $\ell \geq 3$ and $\xi=3$.

Table 3.2. Square-free positive integers $d$ with $3 \leq \ell(d) \leq 12$ (In this table, we rule out $\ell(d)=6$ for $\xi=1$ since $d$ is not a square free positive integer.)

\begin{tabular}{|c|c|c|c|c|c|}
\hline $\boldsymbol{d}$ & $\boldsymbol{\xi}$ & $\boldsymbol{\ell}(\boldsymbol{d})$ & $\boldsymbol{n}_{\boldsymbol{d}}$ & $\boldsymbol{w}_{\boldsymbol{d}}$ & $\boldsymbol{\varepsilon}_{\boldsymbol{d}}$ \\
\hline 8321 & 1 & 3 & 1 & {$[46 ; \overline{9,9,91}]$} & $(7480+82 \sqrt{8321}) / 2$ \\
\hline 2196426007056209 & 1 & 9 & 1 & {$[23433022 ; \overline{9, \ldots 9,46866043}]$} & $\left(\begin{array}{c}2196425574972640+ \\
46866034 \sqrt{2196426007056209}) / 2\end{array}\right.$ \\
\hline 1255340433966050791385 & 1 & 12 & 1 & {$[17715391853 ; \overline{9, \ldots 9,35430783705}]$} & $\left(\begin{array}{c}1276688903859695105858 \\
+35430783696 \sqrt{1255340433966050791385}) / 2\end{array}\right.$ \\
\hline 65137 & 3 & 3 & 3 & {$[128 ; \overline{9,9,255}]$} & $(20928+82 \sqrt{65137}) / 2$ \\
\hline 34590501889 & 3 & 6 & 3 & {$[92993 ; \overline{9, \ldots, 9,185985}]$} & $(11529595730+61992 \sqrt{34590501889}) / 2$ \\
\hline 19767828878503393 & 3 & 9 & 3 & {$[70299056 ; \overline{9, \ldots 9,140598111}]$} & $\left(\begin{array}{c}6589275860750952+ \\
46866034 \sqrt{19767828878503393}\end{array}\right) / 2$ \\
\hline 11298063901774588896481 & 3 & 12 & 3 & {$[53146175549 ; \overline{9, \ldots 9,106292351097}]$} & $\left(\begin{array}{c}3766021300264873946690+ \\
35430783696 \sqrt{11298063901774588896481}\end{array}\right) / 2$ \\
\hline
\end{tabular}


Remark 3. We obtain class number as $h_{d}=10$ for the real quadratic field $\mathbb{Q}(\sqrt{8321})$ and $h_{d}=32$ for the real quadratic field $\mathbb{Q}(\sqrt{65137})$ in the Table 3.2 using the classical Dirichlet class number formula and computer calculations. Besides, we can see that other class numbers are too bigger than class number two by using Proposition 4.1 of reference [8].

Theorem 3.5. Let $d$ be a square free positive integer and $\ell \geq 2$ be a positive integer such that $3 \nmid \ell$. We assume that the parametrization of $d$ is

$$
d=\left(\frac{9+(2 \xi+1) K_{\ell}}{2}\right)^{2}+(2 \xi+1) K_{\ell-1}+1
$$

where $\xi \geq 0$ is a positive integer. Then, following conditions hold:

(1) If $\ell \equiv 1,2,4(\bmod 6)$ and $\xi$ is even positive integer then $d \equiv 2,3(\bmod 4)$.

(2) If $\ell \equiv 5(\bmod 6)$ and $\xi$ is odd positive integer then $d \equiv 2(\bmod 4)$.

Also, in $\mathbb{Q}(\sqrt{d})$ real quadratic fields, we obtain

$$
w_{d}=[\frac{(2 \xi+1) K_{\ell}+9}{2} ; \underbrace{\overline{9,9, \ldots, 9},(2 \xi+1) K_{\ell}+9}_{\ell-1}]
$$

with $\ell=\ell(d)$ for $d \equiv 2,3(\bmod 4)$.

Furthermore, we have the fundamental unit $\varepsilon_{d}$ and coefficients of fundamental unit $t_{d}, u_{d}$ as follows:

$$
\begin{gathered}
\varepsilon_{d}=\left(\frac{9+(2 \xi+1) K_{\ell}}{2} K_{\ell}+K_{\ell-1}\right)+K_{\ell} \sqrt{d}, \\
t_{d}=(2 \xi+1) K_{\ell}^{2}+9 K_{\ell}+2 K_{\ell-1} \text { and } u_{d}=2 K_{\ell} .
\end{gathered}
$$

Proof. If we choose $\ell \equiv 0,3(\bmod 6)$ we obtain that $d$ is not integer because of the parametrization of

$$
d=\left(\frac{9+(2 \xi+1) K_{\ell}}{2}\right)^{2}+(2 \xi+1) K_{\ell-1}+1 .
$$

So, we have to consider that $3 \nmid \ell, \ell \geq 2$ in order to get $d \in Z_{+}$.

(1) If we suppose that $\ell \equiv 1,2(\bmod 6)$, then $K_{\ell} \equiv$ $1(\bmod 4)$. Also, either $K_{\ell-1} \equiv 0(\bmod 4)$ or $K_{\ell-1} \equiv$ $1(\bmod 4)$ hold. By substituting these values into parametrization of $d$ by considering $\xi$ is even positive integer, we obtain $d \equiv 2,3(\bmod 4)$. Moreover, if $\ell \equiv$ $4(\bmod 6)$ and $\xi$ is even positive integer, then $K_{\ell} \equiv$ $3(\bmod 4)$ and $K_{\ell-1} \equiv 2(\bmod 4)$ hold. By substituting these values into parametrization of $d$, then we get $d \equiv 3(\bmod 4)$.

(2) If $\ell \equiv 5(\bmod 6)$ and $\xi$ is odd positive integer then we get $K_{\ell} \equiv 1(\bmod 4)$ and $K_{\ell-1} \equiv 3(\bmod 4)$. Substituting these values into parametrization of $d$ and rearranging, we have $d \equiv 2(\bmod 4)$.

By Lemma 2.3 we get

$$
\begin{aligned}
w_{R}= & \left(\frac{9+(2 \xi+1) K_{\ell}}{2}\right) \\
& +[\frac{9+(2 \xi+1) K_{\ell}}{2} ; \underbrace{9,9, \ldots, 9,(2 \xi+1) K_{\ell}+9}_{\ell-1}]
\end{aligned}
$$

$$
\Rightarrow w_{R}=\left(9+(2 \xi+1) K_{\ell}\right)+\frac{1}{9+\frac{1}{9+\frac{1}{\ddots}}}
$$

So, we have

$$
w_{R}=\left(9+(2 \xi+1) K_{\ell}\right)+\frac{1}{9}+\cdots+\frac{1}{9}+\frac{1}{w_{R}} .
$$

By Lemma 2.3 we obtain

$$
w_{R}=\left(9+(2 \xi+1) K_{\ell}\right)+\frac{K_{\ell-1} w_{R}+K_{\ell-2}}{K_{\ell} w_{R}+K_{\ell-1}} .
$$

Using Definition 2.1 and put $9 K_{\ell}+K_{\ell-1}=$ $K_{\ell+1}$ equation into the above equality, we have

$$
w_{R}^{2}-\left(9+(2 \xi+1) K_{\ell}\right) w_{R}-\left(1+(2 \xi+1) K_{\ell-1}\right)=0 .
$$

This implies that $w_{R}=\left(\frac{9+(2 \xi+1) K_{\ell}}{2}\right)+\sqrt{d}$ since $w_{R}>0$. Let us consider Lemma 2.3 , then we obtain

$$
w_{d}=\sqrt{d}=\left[\frac{9+(2 \xi+1) K_{\ell}}{2} ; \overline{9,9, \ldots, 9,9+(2 \xi+1) K_{\ell}}\right]
$$

and $\ell=\ell(d)$ hold.

Now, we can determine $\varepsilon_{d}, t_{d}$ and $u_{d}$ using Lemma 2.3 as follows:

$$
\varepsilon_{d}=\left(\frac{9+(2 \xi+1) K_{\ell}}{2} K_{\ell}+K_{\ell-1}\right)+K_{\ell} \sqrt{d},
$$

$t_{d}=(2 \xi+1) K_{\ell}^{2}+9 K_{\ell}+2 K_{\ell-1}$ and $u_{d}=2 K_{\ell}$ using the $Q_{i}=K_{i}$ by induction for $\forall i \geq 0$.

Remark 4. We should say that the present paper has got the most general results for such type real quadratic fields. Moreover, we can obtain infinitely many values of $d$ which correspond to new real quadratic fields $\mathbb{Q}(\sqrt{d})$ by using the results.

Corollary 3.6. Let $d$ be square free positive integer and $\ell \geq 2$ be a positive integer satisfying that $\ell \neq \equiv$ $5(\bmod 6), 3+\ell$ Suppose that the parametrization of $d$ is

$$
d=\left(\frac{9+K_{\ell}}{2}\right)^{2}+K_{\ell-1}+1 .
$$

Then, we get $d \equiv 2,3(\bmod 4)$ and

$$
w_{d}=[\frac{9+K_{\ell}}{2} ; \underbrace{\overline{9,9, \ldots, 9}, 9+K_{\ell}}_{\ell-1}]
$$

with $\ell=\ell(d)$. Additionally, we get the fundamental unit $\varepsilon_{d}$, coefficients of fundamental unit $t_{d}, u_{d}$ and Yokoi's invariant $m_{d}$ as follows:

$$
\begin{gathered}
\varepsilon_{d}=\left(\frac{9+K_{\ell}}{2}+\sqrt{d}\right) K_{\ell}+K_{\ell-1}, \\
t_{d}=K_{\ell}^{2}+9 K_{\ell}+K_{\ell-1} \text { and } u_{d}=2 K_{\ell} \\
m_{d}=\left\{\begin{array}{l}
1: \ell=2 \\
3: \ell \geq 4
\end{array}\right.
\end{gathered}
$$

Proof. If we put $\xi=0$ into the Theorem 3.5, then we get 


$$
\begin{gathered}
\varepsilon_{d}=\left(\frac{9+K_{\ell}}{2}+\sqrt{d}\right) K_{\ell}+K_{\ell-1}, \\
t_{d}=K_{\ell}^{2}+9 K_{\ell}+K_{\ell-1} \text { and } u_{d}=2 K_{\ell} . \\
\text { We have to calculate } m_{d}=\llbracket \frac{u_{d}^{2}}{t_{d}} \| \text { defined in the }
\end{gathered}
$$

H.Yokoi's references. If we substitue $t_{d}$ and $u_{d}$ into the $m_{d}$, then we get

$$
m_{d}=\llbracket \frac{u_{d}^{2}}{t_{d}} \rrbracket=\llbracket \frac{4 K_{\ell}^{2}}{K_{\ell}^{2}+9 K_{\ell}+K_{\ell-1}} \rrbracket .
$$

We obtain $m_{d}=1$ for $\ell=2$. For $\ell \geq 4$, we get

$$
4>\frac{4 K_{\ell}^{2}}{K_{\ell}^{2}+9 K_{\ell}+K_{\ell-1}}>3,951
$$

since $K_{\ell}$ is increasing sequence as well as the assumption. Therefore, we obtain $m_{d}=\llbracket \frac{4 K_{\ell}^{2}}{K_{\ell}^{2}+K_{\ell+1}+K_{\ell-1}} \|=3$ for $\ell \geq 4$. For numerical example, let us consider the following Table 3.3 where fundamental unit is $\varepsilon_{d}$, integral basis elemant is $w_{d}$ and Yokoi's invariant is $m_{d}$ for $2 \leq \ell(d) \leq 13$.

Remark 5. The class number is obtained $h_{d}=1$ for the real quadratic field $\mathbb{Q}(\sqrt{83})$ and this field was got with same class number by Mollin in the reference [7] too. The real quadratic field $\mathbb{Q}(\sqrt{142967})$ has got class number $h_{d}=12$ in the Table 3.3 using the classical Dirichlet class number formula and computer calculations. Furthermore, we can not calculate easily class numbers for the other real quadratic fields since they are too bigger than class number two by using Proposition 4.1 of reference [8].

Corollary 3.7. Let $d$ be a square free positive integer and $\ell>1$ be a positive integer satisfying that $\ell \equiv 5(\bmod 6)$. Suppose that the parametrization of $d$ is

$$
d=\left(\frac{9+3 K_{\ell}}{2}\right)^{2}+3 K_{\ell-1}+1
$$

Then, we have $d \equiv 2(\bmod 4)$ and

$$
w_{d}=[\frac{9+3 K_{\ell}}{2} ; \underbrace{\overline{9,9, \ldots, 9}, 9+3 K_{\ell}}_{\ell-1}]
$$

and $\ell=\ell(d)$, Furthermore, we obtain following equalities for $\varepsilon_{d}, t_{d}, u_{d}$ and Yokoi's invariant $m_{d}$.

$$
\varepsilon_{d}=\left(\left(\frac{9+3 K_{\ell}}{2}\right) K_{\ell}+K_{\ell-1}\right)+K_{\ell} \sqrt{d}
$$

$t_{d}=3 K_{\ell}^{2}+9 K_{\ell}+2 K_{\ell-1}$ and $u_{d}=2 K_{\ell}, m_{d}=1$.

Proof. This Corollary is got by substituting $\xi=1$ into the Theorem 3.5.

We assume that $\ell \equiv 5(\bmod 6)$ and $\ell>1$, so we have

$$
\varepsilon_{d}=\left(\left(\frac{9+3 K_{\ell}}{2}\right) K_{\ell}+K_{\ell-1}\right)+K_{\ell} \sqrt{d}
$$

$t_{d}=3 K_{\ell}^{2}+9 K_{\ell}+2 K_{\ell-1}$ and $u_{d}=2 K_{\ell}$

If we substitute $t_{d}$ and $u_{d}$ into the $m_{d}$ and rearranged, then we get

$$
m_{d}=\llbracket \frac{u_{d}^{2}}{t_{d}} \rrbracket=\llbracket \frac{4 K_{\ell}^{2}}{3 K_{\ell}^{2}+9 K_{\ell}+2 K_{\ell-1}} \rrbracket .
$$

\begin{tabular}{|c|c|c|c|c|}
\hline$d$ & $\ell(d)$ & $m_{d}$ & $w_{d}$ & $\varepsilon_{d}$ \\
\hline 83 & 2 & 1 & {$[9 ; \overline{9,18}]$} & $82+9 \sqrt{83}$ \\
\hline 142967 & 4 & 3 & {$[378 ; \overline{9,9,9,756}]$} & $282448+747 \sqrt{142967}$ \\
\hline 79733443634 & 7 & 3 & {$[282371 ; \overline{9,9, \ldots, 9,564742}]$} & $159464283935+564733 \sqrt{79733443634}$ \\
\hline 6616722710135 & 8 & 3 & {$[2572299 ; \overline{9,9, \ldots, 9,5144598}]$} & $13233421704844+5144589 \sqrt{6616722710135}$ \\
\hline 45569206984046339 & 10 & 3 & {$[213469452 ; \overline{9,9, \ldots, 9,426938904}]$} & $\begin{array}{c}91138412000001574+ \\
426938895 \sqrt{45569206984046339}\end{array}$ \\
\hline 26044532297817183855458 & 13 & 3 & {$[1613383184681 ; \overline{9,9, \ldots, 9,322766369362}]$} & $\begin{array}{c}52089064594146488265089+ \\
322766369353 \sqrt{26044532297817183855458}\end{array}$ \\
\hline
\end{tabular}

So, we have

$$
2>4\left(\frac{K_{\ell}^{2}}{3 K_{\ell}^{2}+9 K_{\ell}+2 K_{\ell-1}}\right)>1,332
$$

since $K_{l}$ is increasing sequence. Therefore, we obtain $m_{d}=\llbracket \frac{4 K_{\ell}^{2}}{3 K_{\ell}^{2}+9 K_{\ell}+2 K_{\ell-1}} \rrbracket=1$ for $\ell \equiv 5(\bmod 6)$. To illustrate, let us consider the following Table 3.4 where fundamental unit is $\varepsilon_{d}$, integral basis elemant is $w_{d}$ and Yokoi's invariant is $m_{d}$ for $1<\ell(d) \leq 17$.

Table 3.3. Numerical example for Corollary 3.6.

Table 3.4. Numerical example for Corollary 3.6.

\begin{tabular}{|c|c|c|c|c|}
\hline $\boldsymbol{d}$ & $\boldsymbol{\ell}(\boldsymbol{d})$ & $\boldsymbol{m}_{\boldsymbol{d}}$ & $\boldsymbol{w}_{\boldsymbol{d}}$ & $\boldsymbol{\varepsilon}_{\boldsymbol{d}}$ \\
\hline 104287186 & 5 & 1 & {$[10212 ; \overline{9,9,9,9,20424}]$} & $138986814+6805 \sqrt{104287186}$ \\
\hline 34035254244133659730 & 11 & 1 & {$[5833974138 ; \overline{9,9, \ldots, 9,11667948276}]$} & $\begin{array}{c}22690169478160245177+ \\
3889316089 \sqrt{34035254244133659730}\end{array}$ \\
\hline $\begin{array}{c}11117809286740557533656603 \\
003690\end{array}$ & 17 & 1 & {$[3334337908302120 ; \overline{9,9,6668675816604240}]$} & $\begin{array}{c}7411872857827028108745541593003+ \\
2222891938868077\end{array}$ \\
$\sqrt{111177809286740557533656603003690}$ \\
\hline
\end{tabular}


Corollary 3.8 Let $d$ be square free positive integer and $\ell \geq 2$ be a positive integer satisfying that $\ell \neq \equiv$ $5(\bmod 6), 3+\ell$. We assume that the parametrization of $d$ is

$$
d=\left(\frac{5 K_{\ell}+9}{2}\right)^{2}+5 K_{\ell-1}+1
$$

Then, we get $d \equiv 2,3(\bmod 4)$ and

$$
w_{d}=[\frac{5 K_{\ell}+9}{2} ; \underbrace{\overline{9,9, \ldots, 9}, 9+5 K_{\ell}}_{\ell-1}]
$$

with $\ell=\ell(d)$. Besides, we obtain the fundamental unit $\varepsilon_{d}$, coefficients of fundamental unit $t_{d}, u_{d}$ and Yokoi's invariant $n_{d}$ as follows:

$$
\varepsilon_{d}=\left(\frac{5 K_{\ell}+9}{2}+\sqrt{d}\right) K_{\ell}+K_{\ell-1},
$$

$t_{d}=5 K_{\ell}^{2}+9 K_{\ell}+2 K_{\ell-1}$ and $u_{d}=2 K_{\ell}, n_{d}=1$.

Proof. We have this corollary by using Theorem 3.5 for $\xi=2$. It is just enough to calculate $n_{d}$ defined as $n_{d}=\llbracket \frac{t_{d}}{u_{d}^{2}} \rrbracket$. If we substitue $t_{d}$ and $u_{d}$ into the $n_{d}$, then we get

$$
\begin{aligned}
n_{d} & =\llbracket \frac{t_{d}}{u_{d}^{2}} \rrbracket \\
& =\llbracket \frac{5 K_{\ell}^{2}+9 K_{\ell}+2 K_{\ell-1}}{4 K_{\ell}^{2}} \rrbracket \\
& =1+\llbracket \frac{1}{4}+\frac{9}{4 K_{\ell}}+\frac{K_{\ell-1}}{2 K_{\ell}^{2}} \rrbracket .
\end{aligned}
$$

If we consider that $K_{\ell}$ is increasing sequence, we calculate following inequality

$$
0<\frac{K_{\ell}^{2}+9 K_{\ell}+2 K_{\ell-1}}{4 K_{\ell}^{2}}<0,253
$$

for $\ell \geq 2$.

Hence, we obtain $n_{d}=\llbracket \frac{5 K_{\ell}^{2}+9 K_{\ell}+2 K_{\ell-1}}{4 K_{\ell}^{2}} \rrbracket=1$ for $\ell \geq 2$.

For numerical example, let us consider the following table where the fundamental unit is $\varepsilon_{d}$, integral basis elemant is $w_{d}$ and and Yokoi's invariant is $n_{d}$ for $2 \leq \ell(d) \leq 13$.

Table 3.5. Numerical example for Corollary 3.7 (In the following table, we rule out $\ell(d)=2,10,13$ since $d$ is not a square free positive integer in these periods)

\begin{tabular}{|c|c|c|c|c|}
\hline $\boldsymbol{d}$ & $\boldsymbol{\ell}(\boldsymbol{d})$ & $\boldsymbol{n}_{\boldsymbol{d}}$ & $\boldsymbol{w}_{\boldsymbol{d}}$ & $\boldsymbol{\varepsilon}_{\boldsymbol{d}}$ \\
\hline 3504795 & 4 & 1 & {$[1872 ; \overline{9,9,9,3744}]$} & $1398466+747 \sqrt{3504795}$ \\
\hline 1993284024530 & 7 & 1 & {$[1411837 ; \overline{9,9, \ldots, 9,2823674}]$} & $797311006513+564733 \sqrt{1993284024530}$ \\
\hline 165417593445195 & 8 & 1 & {$[12861477 ; \overline{9,9, \ldots, 9,25722954}]$} & $66167013662686+5144589 \sqrt{1654175934445195}$ \\
\hline
\end{tabular}

Remark 6. The class number is $h_{d}=128$ for the real quadratic field $\mathbb{Q}(\sqrt{3504795})$ in the Table 3.5 using the classical Dirichlet class number formula and computer calculations. Furthermore, we can not calculate easily other class numbers since they are too bigger than class number two by using Proposition 4.1 of reference [8].

\section{Conclusion}

In this paper, we introduced the notion of real quadratic field structures such as continued fraction expansions, fundamental unit and Yokoi invariants where $d$ is square free positive integer. We established general interesting and significant results for that. Results obtained in this paper provide us a useful and practical method so as to rapidly determine continued fraction expansion of $w_{d}$ fundamental unit $\varepsilon_{d}$ and and Yokoi invariants $n_{d}$ for such real quadratic number fields. There are some authors work on structure of the real quadratic number fields, but the results in this paper are new and more general for such types of real quadratic fields.

Findings in this paper will help the researchers to enhance and promote their studies on quadratic fields to carry out a general framework for their applications in life.

\section{References}

[1] Benamar H., Chandoul A. and Mkaouar M. (2015). On the Continued Fraction Expansion of Fixed Period in Finite Fields, Canad. Math. Bull. 58, 704-712.

[2] Clemens L. E., Merill K. D., Roeder D. W. (1995). Continues fractions and series, J. Number Theory 54, 309-317.

[3] Elezovi'c N.(1997). A note on continued fractions of quadratic irrationals, Math. Commun. 2, 27-33.

[4] Halter-Koch F.,(1991). Continued fractions of given symmetric period. Fibonacci Quart., 29(4), 298-303.

[5] Kawamoto F. and Tomita K. (2008). Continued fraction and certain real quadratic fields of minimal type, J.Math.Soc. Japan, 60, 865-903.

[6] Louboutin S. (1988). Continued Fraction and Real Quadratic Fields, J.Number Theory, 30, 167-176, 1988.

[7] Mollin R. A. (1996). Quadratics, CRC Press, Boca Rato, FL, 399p.

[8] Mollin R. A., Williams, H.C. (1992). On Real Quadratic Fields of Class Number Two, Math. of Comp. 59(200), 625-632.

[9] Olds C. D. (1963). Continued Functions, New York, Random House, $170 \mathrm{p}$.

[10] Özer Ö. (2016). On Real Quadratic Number Fields Related With Specific Type of Continued Fractions, Journal of Analysis and Number Theory, 4(2), 85-90.

[11] Özer Ö. (2016). Notes On Especial Continued Fraction Expansions and Real Quadratic Number Fields, Kirklareli University Journal of Engineering and Science, 2(1), 74-89.

[12] Perron O. (1950). Die Lehre von den Kettenbrichen, New York: Chelsea, Reprint from Teubner Leipzig, $200 \mathrm{p}$.

[13] Sasaki R. (1986). A characterization of certain real quadratic fields, Proc. Japan Acad., 62, Ser. A, No. 3, 97-100. 
[14] Sierpinski W. (1964). Elementary Theory of Numbers, Warsaw: Monografi Matematyczne.

[15] Tomita, K., 1995. Explicit representation of fundamental units of some quadratic fields, Proceeding Japan Academia, 71, Ser. A, No. 2, 41-43.

[16] Tomita, K. and Yamamuro K., 2002. Lower bounds for fundamental units of real quadratic fields, Nagoya Mathematical Journal,166, 29-37.

[17] Williams, K. S., and Buck, N., 1994. Comparison of the lengths of the continued fractions of $l(\sqrt{d})$ and $l\left(\frac{1+\sqrt{d}}{2}\right)$. Proceeding American Mathematical Society, 120(4), 995-1002, 1994.
[18] Yokoi H. (1990). The fundamental unit and class number one problem of real quadratic fields with prime discriminant, Nagoya Math. J., 120, 51-59.

[19] Yokoi H. (1991). The fundamental unit and bounds for class numbers of real quadratic fields, Nagoya Math. J., 124, 181-197.

[20] Yokoi H., 1993. A note on class number one problem for real quadratic fields. Proc. Japan Acad., 69, Ser. A, 22-26.

[21] Yokoi H, 1993. New invariants and class number problem in real quadratic fields. Nagoya Math. J., 132, 175-197.

[22] Zhang, Z. and Yue, Q., 2014. Fundamental units of real quadratic fields of odd class number. Journal of Number Theory 137, 122-129. 